\title{
Changes of ongoing activity in Cebus monkey perirhinal cortex correlate with behavioral performance
}

B. Lima, M. Fiorani and R. Gattass
Departamento de Neurobiologia, Instituto de Biofísica Carlos Chagas Filho, Universidade Federal do Rio de Janeiro, Rio de Janeiro, RJ, Brasil

\section{Correspondence \\ R. Gattass \\ Departamento de Neurobiologia \\ Instituto de Biofísica Carlos \\ Chagas Filho, BI. G, CCS, UFRJ \\ 21941-900 Rio de Janeiro, RJ \\ Brasil \\ Fax: +55-21-2280-8193 \\ E-mail: rgattass@biof.ufrj.br}

Research supported by CNPq, PRONEX, FUJB, and FAPERJ.

Received December 9, 2003

Accepted October 6, 2004

\begin{abstract}
A Cebus apella monkey weighing $4 \mathrm{~kg}$ was trained in a saccadic eye movement task and while the animal performed the task we recorded the extracellular activity of perirhinal cortical neurons. Although the task was very simple and maintained at a constant level of difficulty, we observed considerable changes in the performance of the monkey within each experimental session. The behavioral states responsible for such variation may be related to arousal, motivation or attention of the animal while engaged in the task. In approximately $20 \%$ (16/82) of the units recorded, long-term direct or inverse correlations could be demonstrated between the monkey's behavioral state and the cells' ongoing activity (independent of the visual stimulation or of the specific behavior along a trial). The perirhinal cortex and other medial temporal structures have long been associated with normal memory function. The data presented here were interpreted in terms of recent reports focusing on the subcortical afferents to temporal lobe structures and their possible role in controlling arousal, motivation, or attention.
\end{abstract}

Key words

- Perirhinal cortex

- Behavioral states

- Motivation

- Cebus monkeys

.....................
Medial temporal lobe structures have long been associated with normal memory function (1). Lesion $(2,3)$ and neurophysiological (4-6) studies have attributed a critical role in object recognition memory to the perirhinal cortex (7). The general assumption has been that the neural substrate subserving the mnemonic process is located in these cortical structures. Recent study (8) on monkeys has addressed this issue directly. The subcortical afferents to the medial temporal lobe were interrupted while the cortical tissue was left intact. When the lesioned animals were tested behaviorally they exhibited severe memory impairment similar to that classically ob- served in animals with lesions in medial temporal lobe areas. These data strongly suggest that widespread modulatory projections of subcortical origin affecting brain state and possibly arousal, attention and/or motivation could be essential for memory formation. Additionally, the data suggested that the interruption of these projections to medial temporal lobe structures could, by itself, reproduce the dense amnesia observed in the classical ablation studies.

In parallel, another set of recent studies (9-11) has focused on the role of the rhinal cortex in associating visual stimuli with their motivational significance. In these studies 
motivation was manipulated by varying the amount of trials the monkey had to execute correctly before receiving the reward. The reward schedule applied to the trial (i.e., the proximity of the reward) was indicated to the animal by the brightness or the length of a bar. Normal animals automatically adjusted their behavior to the reward schedule. The animals performed better in trials in which the reward was about to happen and progressively worse in trials with longer reward schedules. This behavioral adjustment to the reward schedule was impaired after surgical ablation of the rhinal cortex (10), a finding supported by data obtained by electrophysiology. In a similar experimental design (9), the activity of perirhinal cortical neurons carried information about the progress of the animal through the reward schedule, while neurons from the anterior lateral temporal cortical area did not.

In the present study, we investigated if changes of behavioral states, as measured by task performance, correlated with ongoing neuronal activity in the middle temporal lobe (perirhinal cortex) of a primate. All experimental procedures were conducted in accordance with the guidelines for care and use of laboratory animals (CAUAP) of the Institute of Biophysics Carlos Chagas Filho. One Cebus apella monkey weighing $4 \mathrm{~kg}$ was used in this study. The methods of anesthesia, single unit recording and histological processing have been described in detail elsewhere $(12,13)$. Briefly, the animal was implanted with a recording chamber and a head bolt under sterile conditions, and a scleral search coil was also implanted to monitor eye position (14). The results reported here were obtained as part of a project devoted to the study of spatial selectivity of perirhinal neurons (15).

The monkey was trained to gaze a fixation point (FP) roughly in the center of a $21 "$ computer screen and to make a saccadic eye movement to a peripheral target. The fixation window was $3.5 \times 3.5^{\circ}$ large and cen- tered on the FP or on the target. The animal had to hold fixation on the FP $\left(0.3 \times 0.3^{\circ}\right)$ for $500 \mathrm{~ms}$. The FP was turned off at the same time as a peripheral target appeared. The stimulus used as target was a colored disc or a picture of the face of a monkey ranging from 1 to $8^{\circ}$ in its longest dimension. The animal had to make a saccadic eye movement to the target and gaze it for at least 650 $\mathrm{ms}$ to receive a liquid reward (fruit juice). The FP could appear in one of two positions and the target could appear in one of eight positions. All conditions were presented randomly in blocks. To successfully complete an experimental session, the monkey had to perform 480 correct trials. Not all experimental sessions were completed. The monkey was in an initial period of training and was not being submitted to a severe water deprivation protocol.

Using 1.2-M $\Omega$ tungsten microelectrodes, we recorded extracellular potentials from 82 neurons in the perirhinal cortex isolated with the aid of a spike sorter (Signal Processing System, SPS, Prospect, Australia). No attempt was made to isolate cells that responded to the visual stimulus. All trials (correct and incorrect) of a behavioral session were analyzed. For each trial, two behavioral parameters and the neuronal activity from two periods were considered. The behavioral parameters used were the correct and incorrect status of the trial and the speed of saccade onset (saccade speed) to the target. The discrete correct or incorrect status of the trial was transformed to a smoothed percent correct curve using a Gaussian sliding window. Therefore, the smoothed percent correct value for each trial considers the weighted contribution from flanking trials. To permit comparisons, the same smoothing procedure was applied to the curve of the saccade speed and to the curves of neuronal activity. Neuronal activity from two periods was computed for each trial: a) an average of the activity during the whole trial, which was subjected to visual stimulus modulation, and b) an average 
of the spontaneous activity, which was independent of visual stimulation. These four curves were plotted together and coincident peaks and troughs were determined by visual inspection. We studied the correlation of the behavioral states as measured by task performance (percent correct or saccade speed), with the neuronal activity (firing rate) in the middle temporal lobe (perirhinal cortex) by the least square method (linear regression). We also studied the correlation of the direction of change in the firing rate and the performance of the animals. We computed the statistical significance (Pearson chi-square) of the incidence of increase or decrease of firing rate with the decrease or increase of the animal's performance, using the sign of the first derivative of both parameters.

The two behavioral parameters considered, percent correct and speed of saccade to target, correlated consistently with each other in only $10 \%(4 / 41)$ of the experimental ses- sions. When this occurred, only direct correlations were observed between the two. Thus, a better performance generally reflected a higher percent correct along with a higher speed of saccade to target.

For $20 \%$ of the neurons recorded (16/ 82 ), correlations were observed between the neuronal activity and the behavioral parameters during one or more periods of the experimental session. For $56 \%$ of these units (9/16), a direct correlation was observed between activity and behavior. Therefore, an increase in neuronal activity occurred when the monkey was performing better as measured by a higher percent correct (7/9) or by both higher percent correct and faster saccade to the target (2/9). An example of a neuron in which a direct correlation was observed between neuronal activity and behavior is shown in Figure 1A. This is an example of a session in which the monkey was performing very poorly, with periods during which the animal completely stopped
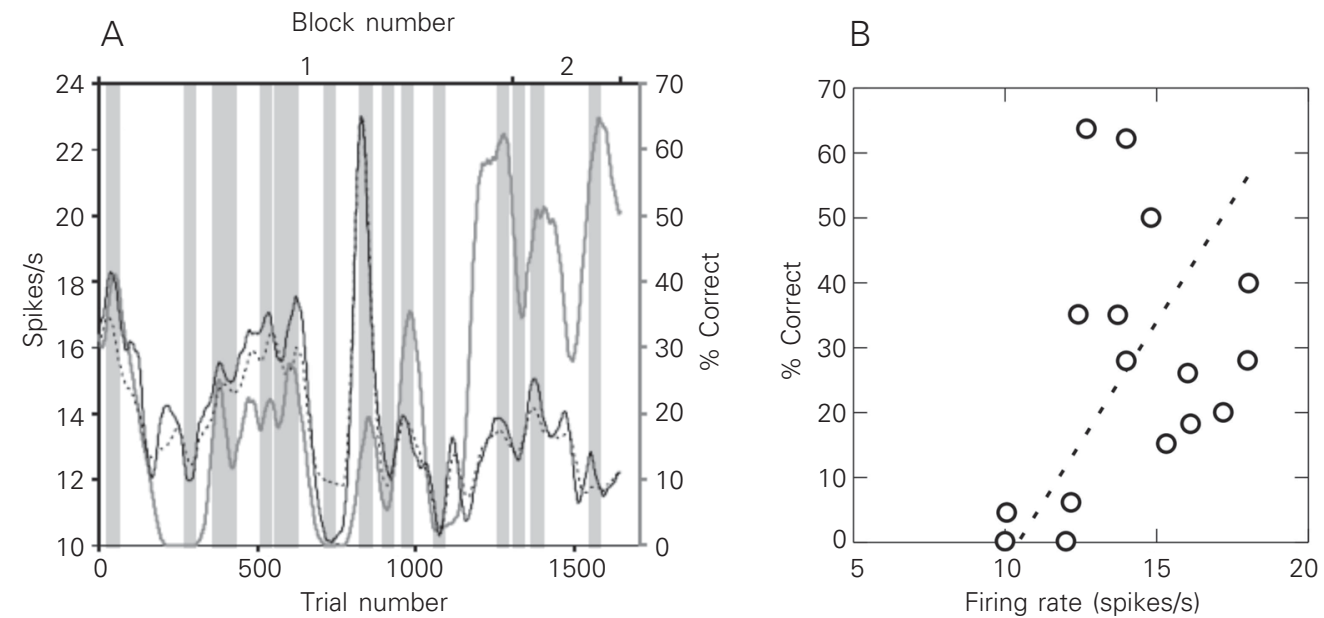

Figure 1. A, Activity of a perirhinal neuron whose firing rate shows a direct correlation with the monkey's behavioral performance. Smoothed firing rate and percent correct performance are plotted against trial and block number. The continuous and dotted black lines indicate the spontaneous and whole-trial activity, respectively, with the scale on the left vertical axis in spikes per second. The continuous gray line indicates the monkey's behavioral performance with the scale on the right vertical axis, as \% correct. All three curves were Gaussian smoothed using the same sliding window. Vertical gray bars, whose width corresponds to approximately 25 trials, were positioned to indicate the coincidence of peaks or troughs between neuronal activity and behavioral performance. Observe the coincidence between these two parameters at the position of the peaks and troughs, independent of their amplitude. The whole trial firing rate and the spontaneous activity correlated in the same way with behavioral performance. $B$, Lack of correlation $(P=0.67)$ between neuronal activity (spike rate) and behavioral performance (\% correct). The dashed line represents the regression line determined by the least square method. 
responding. When the monkey restarted working, however, a build-up of neuronal activity was observed. It is important to note that the correlation between neuronal activity and behavior was mainly due to the coincidence of the peaks or troughs but not to their amplitudes. The least square method showed that the correlation between the firing rate and the behavioral performance was not statistically significant.

However, the relationship between behavior and neuronal activity was not restricted only to direct correlations. Interestingly, for $44 \%$ of the modulated units (7/16), an inverse correlation was observed between behavior and neuronal activity. In these cases, a decrease in neuronal activity was observed when the monkey was performing better as measured by a higher percent correct (4/7), a faster saccade to the target $(1 / 7)$ or both $(2 / 7)$.

The neuronal activity of a neuron with an inverse correlation between neuronal activity and behavior is shown in Figure 2. In the illustrated experimental session, the monkey showed an intermediate behavioral performance but did not completely stop working during any period. Contrary to the example shown in Figure 1, a coupling between neuronal activity and behavior only began after more than 200 trials had been performed. Curiously, the beginning of the coupling

Figure 2. A, Activity of a perirhinal neuron whose firing rate shows an inverse correlation with the monkey's behavioral performance. Conventions are the same as in Figure 1. The changes in the spontaneous and in the whole trial activities for this cell were very similar to each other. $B$, The regression line, fitted by the least square method $(P=0.04)$, shows a significant linear correlation, for both the slope and the intercept, between the neuronal activity (spike rate) and the behavioral performance (\% correct, \%C). $F R=$ firing rate

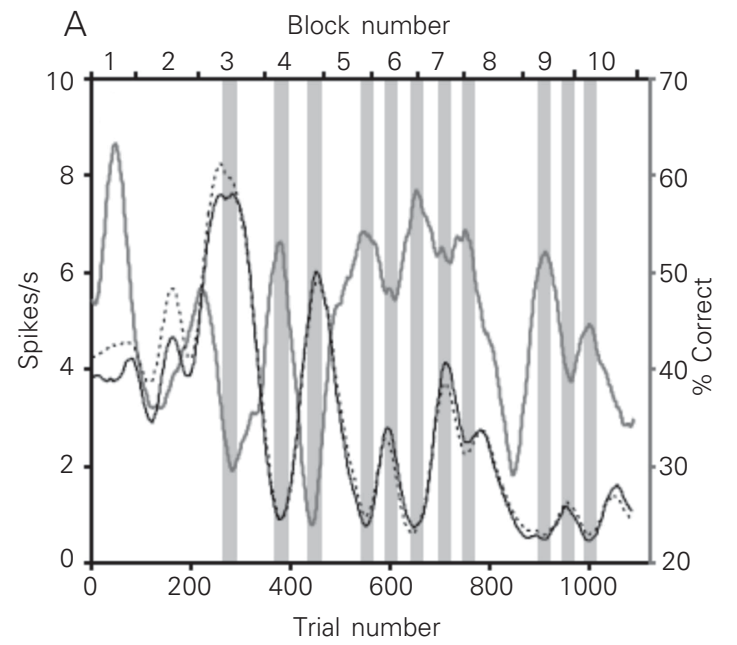

B

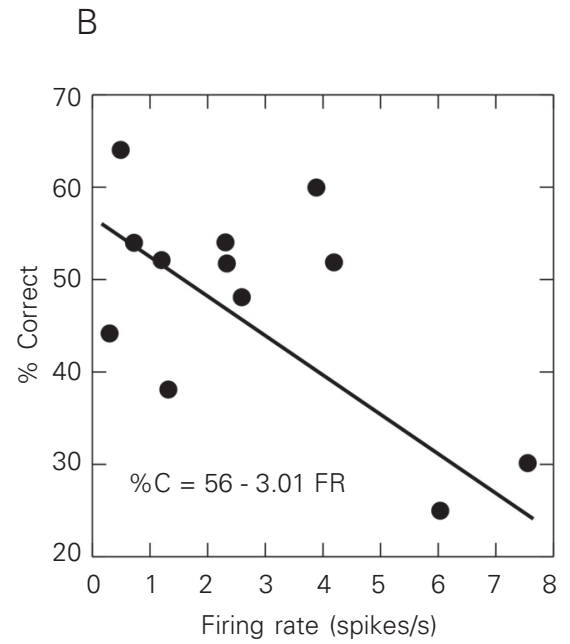

coincided with the beginning of the period during which the whole-trial and spontaneous activities matched. Again, the correlation between behavior and neuronal activity was reflected more by the coincidence of peaks and troughs than by their amplitudes. When present, direct or inverse correlations of neuronal activity generally occurred with the behavioral parameter "percent-correct". In a small sample of the units (4\%) there was a significant direct or inverse correlation between the spike rate and the behavioral performance (observed in Figure 2 but not in Figure 1) as revealed by the least square method. It should be pointed out that the "least square" may not be the most adequate test since it is sensitive to the amplitude of the peaks and troughs. By visual inspection of the data it is clear that correlations between spike rate and behavior are essentially present in the position of the peaks and troughs rather than in their amplitude. Using these criteria, $20 \%$ of the perirhinal neurons showed correlation of their spike rate with behavior (Pearson chi-square $=7.08, \mathrm{P}=$ 0.008 for direct correlations and Pearson chi-square $=7.13, \mathrm{P}=0.008$ for the indirect correlations). When neuronal activity was correlated with the saccade speed to target, correlation with percent correct was nearly always also present.

The present results show that the firing 
rate of individual neurons in the monkey perirhinal cortex can reflect performance in the behavioral task the animal is engaged in. The causes of variation in performance due to changes in the animal's behavioral state were probably related to factors such as arousal, attention and motivation. Curiously, the modulatory systems that affect behavior also seem to affect the ongoing activity of neurons in the perirhinal cortex, an area in the medial temporal lobe classically associated with mnemonic processes. Therefore, it is reasonable to speculate that modulatory systems, probably of subcortical origin, modulate the global activity of medial temporal lobe structures and ultimately the memory formation processes taking place in this region.

The interaction of the sensory and motor systems with the brain's modulatory systems concerned with reward, attention, and motivation is fundamental for the understanding of learning and memory storage. The effect we observed may well have some physiological correlate with the effects of lesioning subcortical afferents to the medial temporal lobe described by Gaffan and collaborators (8). Widely distributed projections of this type can account for fluctuations of neuronal firing independent of sensory stimulation as we observed in the present study. These modulatory projections may have a great influence in determining the cell's membrane potential and its threshold for firing. The excitability of a neuron obviously plays a major role in the Hebbian rules of synaptic plasticity, a possible neural mechanism for learning and memory formation (16).

\section{Acknowledgments}

We wish to thank Edil Saturato da Silva Filho and Theresa Monteiro for skillful technical assistance, and Paulo Coutinho and Gervasio Coutinho for animal care.

\section{References}

1. Milner B (1972). Disorders of learning and memory after temporal lobe lesions in man. Neuropsychologia, 19: 421-446.

2. Zola-Morgan SM, Squire LR, Amaral DG \& Suzuki W (1989). Lesions of perirhinal and parahippocampal cortex that spare the amygdala and hippocampal formation produce severe memory impairments. Journal of Neuroscience, 9: 4355-4370.

3. Meunier M, Bachevalier J, Mishkin M \& Murray E (1993). Effects on visual recognition of combined and separate ablations of the entorhinal and perirhinal cortex in rhesus monkeys. Journal of Neuroscience, 13: 5418-5432.

4. Brown MW, Wilson FAW \& Riches IP (1987). Neuronal evidence that inferomedial temporal cortex is more important than hippocampus in certain processes underlying recognition memory. Brain Research, 409: 158-162.

5. Miller EK, Li L \& Desimone R (1991). A neural mechanism for working and recognition memory in inferior temporal cortex. Science, 254: 1377-1379.

6. Miller EK \& Desimone R (1994). Parallel neuronal mechanisms for short-term memory. Science, 263: 520-523.

7. Murray E \& Bussey TJ (1999). Perceptual-mnemonic functions of the perirhinal cortex. Trends in Cognitive Sciences, 3: 142-151.

8. Gaffan D, Parker A \& Easton A (2001). Dense amnesia in the monkey after transection of fornix, amygdala and anterior temporal stem. Neuropsychologia, 39: 51-70.
9. Liu Z \& Richmond BJ (2000). Response differences in monkey TE and perirhinal cortex: stimulus association related to reward schedules. Journal of Neurophysiology, 83: 1677-1692.

10. Liu Z, Murray E \& Richmond BJ (2000). Learning motivational significance of visual cues for reward schedules requires rhinal cortex. Nature Neuroscience, 3: 1307-1315.

11. Murray EA \& Richmond B (2001). Role of perirhinal cortex in object perception, memory, and associations. Current Opinion in Neurobiology, 11: 188-193.

12. Gallyas F (1979). Silver staining of myelin by means of physical development. Neurological Research, 1: 203-209.

13. Gattass R \& Gross C (1981). Visual topography of the striate projection zone in the posterior superior temporal sulcus (MT) of the macaque. Journal of Neurophysiology, 46: 621-638.

14. Judge SJ, Richmond BJ \& Chu FC (1980). Implantation of magnetic search coils for measurement of eye position: an improved method. Vision Research, 20: 535-538.

15. Lima B, Fiorani M \& Gattass R (2003). Modulation by context of a scene in the monkey anterior inferotemporal cortex during a saccadic eye movement task. Annals of the Brazilian Academy of Sciences, 75: 71-76.

16. Hebb DO (1949). The Organization of Behavior. John Wiley \& Sons, New York. 